\title{
Losas de concreto reforzadas con acero inoxidable de desecho
}

\section{Reinforced concrete slabs with stainless steel waste}

\author{
Jorge Alberto Pérez González*1
}

* Universidad Autónoma de San Luis Potosí. MÉXICO

Fecha de recepción: 02/ 07/ 2008 Fecha de aceptación: 31/ 07/ 2008 PAG. $72-81$

Resumen

\begin{abstract}
Se muestran los resultados de un programa experimental que explora la reutilización de materiales de desecho industrial (específicamente láminas de acero inoxidable producto de la acuñación de moneda) como refuerzo en losas de concreto. Para ello se elaboraron 23 especímenes tipo viga-losa a escala natural con dicho refuerzo a fin de determinar su comportamiento en términos de resistencia, ductilidad y formas de falla; el análisis experimental de modelos ensayados con diferentes cantidades de refuerzo muestra que en algunos casos, bajo ciertas condiciones de cuantías y colocación del mismo, es posible alcanzar capacidades de carga y formas de falla similares a las de especímenes de control con refuerzo tradicional. En base a estos resultados se concluye en la factibilidad de utilizar el material arriba descrito como refuerzo alternativo en elementos estructurales de concreto, tratando de encontrar alternativas más económicas en la construcción de vivienda popular.
\end{abstract}

Palabras Clave: Concreto reforzado, acero inoxidable, comportamiento, resistencia, formas de falla

Abstract

The results from an experimental program that explores the use of industrial waste materials (specifically sheets of stainless steel that result of the currency coinage) as reinforcement for concrete slabs are presented. Twenty three full size beam-deck specimens were built in order to measure resistance, ductility and failure modes; the experimental analysis in models with reinforcement in different quantities shows that in some cases, under certain quantities and location, it is possible to reach similar load capacities and failure modes as specimens with traditional reinforcement. Based on these results, it is concluded the feasibility of using the material described above as alternative reinforcement in structural concrete elements, as an economic option in the construction of social housing.

Keywords: Reinforced concrete, stainless steel, performance, strength, failure modes

\section{Introducción}

La corrosión del acero de refuerzo es la causa mas importante del deterioro prematuro de las estructuras de concreto armado (Li et al., 2005) y, a diferencia de las acciones correctivas como el uso de inhibidores de corrosión o cubiertas epóxicas, el utilizar acero inoxidable como refuerzo se considera una acción preventiva que trae como resultado una esperanza de vida útil de más de 75 años (Medina-Romero, 2006) y una reducción de los costos de mantenimiento; un estudio realizado en 2007 (Baltasar et al., 2007) sobre barras de refuerzo de acero inoxidable embebidas en especímenes de concreto expuestos durante 66 semanas a la acción de cloruros y sulfatos, demostró velocidades de corrosión de 30 a 150

\footnotetext{
${ }^{1}$ Autor de correspondencia / Corresponding author:

Av. Dr. Manuel Nava No 8, Zona U niversitaria, San Luis Potosí, S.L.P.

México. C.P. 78290.

Teléfono: (444) 826-23-30, E-mail: perezjor@uaslp.mx
}

veces menor que el refuerzo convencional, dependiendo de la relación agua cemento. Por esta razón, y por su relativamente mayor costo en comparación con el del acero al carbono, históricamente el uso estructural del acero inoxidable se ha limitado al refuerzo de estructuras de concreto particularmente expuestas a ambientes agresivos, tales como estructuras marítimas o de difícil mantenimiento por los altos volúmenes de tráfico como es el caso de puentes carreteros o ferroviarios; aún en estos casos, el acero inoxidable es utilizado únicamente en los elementos estructurales mayormente expuestos como sustitución parcial de la varilla ordinaria (H unkeler, 2000).

El acero inoxidable como armadura de refuerzo del concreto armado se empleó por primera vez en la 
erección del muelle de Puerto Progreso, en el estado mexicano de Yucatán, en las costas del Golfo de México. Construido entre los años 1937 y 1941 por la empresa danesa Christiani and Nielsen, el éxito de su diseño radica en la minimización del uso del acero de refuerzo utilizando concreto masivo para la sub y superestructuras, empleando tan solo de manera importante barras de acero inoxidable de $30 \mathrm{~mm}$ en cantidad de 220 tn como refuerzo de los cabezales de la subestructura. En el año 2001 se llevó al cabo una exhaustiva inspección por durabilidad de la estructura del muelle en 100 de los 146 arcos que conforman el viaducto, que incluyó la medición de recubrimientos, obtención de muestras para medición de cloruros, resistividad y potenciales y velocidades de corrosión (Moreno et al., 2004); los análisis revelaron concentraciones importantes de cloruros del orden del $2 \%$ por peso del concreto, mismos que en la literatura pronostican un estado de iniciación de la corrosión (CastroBorges et al., 2002), y por otro lado, las mediciones de potenciales y velocidades de corrosión sugirieron un estado activo en las barras de acero inoxidable. En inspecciones posteriores realizadas durante 2003 y 2004 se observó la presencia de signos de corrosión en los ganchos de anclaje de las barras, en un principio atribuidos a los esfuerzos residuales inducidos durante las operaciones de doblado, sin embargo, en un estudio realizado en 2006 por el Instituto M exicano del Transporte, se obtuvieron diferencias marginales en el comportamiento electroquímico entre barras rectas de acero inoxidable y barras que presentan esfuerzos residuales por doblado (Torres-Acosta et al., 2006) y, aunque el concreto presenta una alta contaminación por cloruros, los resultados permitieron comprobar la inexistencia de corrosión, atribuyendo su estado incipiente a la antigüedad de la estructura de mas de 65 años y a la fuerte exposición al ambiente marino.

No existen otros usos significativos de armadura de acero inoxidable documentados durante los 40 años posteriores a la construcción del muelle de Progreso. En 1984 el Departamento del Transporte de Michigan utilizó barras de acero inoxidable en un puente al norte de Detroit; a principios de la década de 1990 se emplearon también en la restauración del patio delantero del Sydney 0 pera House, y, durante la misma década, se construyeron algunos puentes en los estados norteamericanos de $\mathrm{O}$ regon y $\mathrm{N} \mathrm{ew}$ Jersey, así como en O ntario, Canadá, y un puerto en los Emiratos Árabes Unidos. De manera excepcional, en el puente Haynes en O regon, construido en 2002, se emplearon mas de 400 tn de refuerzo de acero inoxidable (Torres-Acosta et al., 2006).

A pesar del poco difundido empleo del acero inoxidable como refuerzo en estructuras de concreto, a principios de la década de 1970 el Building Research Establishment inició un ambicioso programa de investigación que duraría mas de 20 años para determinar la resistencia a la corrosión de diferentes materiales para el armado, incluyendo el acero inoxidable (MedinaRomero, 2006); sin embargo, aún cuando los estudios revelaron un excelente comportamiento del material, este no fue adoptado por códigos de diseño de manera significativa.

En 1998 la Concrete Society publicó un informe técnico sobre el uso puntual de armaduras de acero inoxidable (Concrete Society, 1998), mismo que habría de ser tomado como punto de partida para que en ese mismo año la Highways Agency, principal responsable del diseño, mantenimiento y restauración del sistema carretero del Reino U nido, iniciara su propio programa de investigación que daría como resultado la publicación en 2002 de una nota departamental, la BA 84/02, "U se of Stainless Steel Reinforcement in Highway Structures." (The Highways Agency, 2002), dentro de su Manual de Diseño de Carreteras y Puentes para el uso de refuerzo de acero inoxidable en estructuras de concreto. De manera similar, la British Standards Institution, la oficina nacional de normalización del Reino U nido, publicaría en septiembre de 2001 la norma BS 6744:2001, "Stainless steel bars for the reinforcement of and use in concrete requirements and test methods" (The British Standards Institution, 2001), primera y única normativa nacional para el armado de acero inoxidable en Europa, y que habría de sustituir a la primera versión de 1986. En Estados U nidos, la American Society for Testing Materials publicaría por primera vez en 1996 la norma A955 "Standard Specification for Deformed and Plain Stainless-Steel Bars for Concrete Reinforcement" (ASTM, 1996) para el uso de barras de acero inoxidable como refuerzo en estructuras de concreto susceptibles a la corrosión o en las que se requiere cierto grado de permeabilidad magnética, norma que sería revisada en sucesivas actualizaciones hasta desembocar en su versión de 2007 actualmente en vigor. No obstante todos los esfuerzos que en materia de investigación y normatividad se han realizado en los últimos años y que el uso en la industria de la construcción del acero inoxidable es cada vez más habitual, lo cierto es que su empleo como refuerzo principal en estructuras de concreto es aún muy limitado comparativamente respecto del acero al carbono; por un lado, la todavía escasa normatividad y la reducida gama de productos disponibles, y por el otro el alto costo del material, colocan a aquél en clara desventaja respecto de este (los resultados 
de un programa de investigación realizado en 1999 por las administraciones de carreteras de las provincias suizas reveló como principal obstáculo para su uso los altos costos del material)(Hunkeler, 2000).

U na de las principales aplicaciones del acero inoxidable en México consiste en la acuñación de moneda del sistema monetario nacional y de otros países centro y sudamericanos. A partir del año de 1983 se establece en el parque industrial de la ciudad de San Luis Potosí la planta de Productora de Cospeles S.A. de C.V., empresa filial de Casa de Moneda de México, constituida en 1981, y proyectada para la fabricación de cospeles o discos metálicos para la acuñación de moneda. La materia prima utilizada en la fabricación del cospel consiste en láminas de acero inoxidable de distintos espesores; dichas láminas, una vez troqueladas para la obtención del producto final, se convierten en material de desecho cuya mayor parte es vendido a compañías fundidoras, muy por debajo del precio de mercado, para su posterior reutilización en productos con menores requerimientos de calidad del material. Algunos estudios experimentales previos, que sin embargo aún no han sido debidamente documentados, demuestran que es posible utilizar dicho material de desecho a manera de refuerzo en losas de concreto de claros pequeños como las empleadas en viviendas de tipo popular, a precios competitivos respecto al refuerzo convencional a base de malla de alambre electrosoldado. La Figura 1 muestra este material del que se obtuvieron cospeles para la acuñación de moneda de un peso del sistema monetario mexicano.

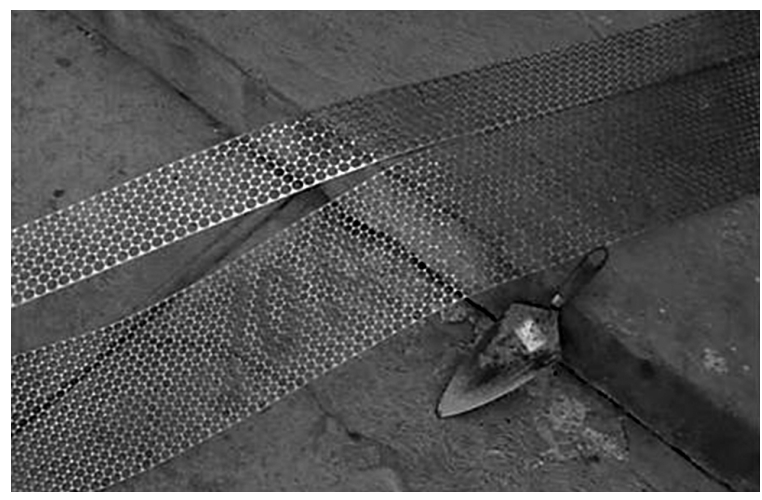

Figura 1. Material de desecho de acero inoxidable

Se muestran aquí los resultados de un análisis experimental efectuado en modelos a escala natural reforzados con el material postulado. La presentación se compone básicamente de tres partes, la primera de las cuales se refiere a la descripción de las propiedades de los materiales a utilizar en las pruebas de los especímenes, acero al carbono, acero inoxidable y concreto hidráulico, así como las pruebas efectuadas en dichos materiales; la segunda parte consiste en la caracterización del programa experimental propiamente; finalmente, la tercera parte del trabajo corresponde a la discusión e interpretación de los resultados obtenidos, así como su comparabilidad con el comportamiento de especímenes de control fabricados con refuerzo convencional, para llegar finalmente a concluir en la factibilidad del uso del acero inoxidable como refuerzo alternativo.

\section{Materiales utilizados}

\subsection{Acero al carbono en especímenes de control}

Para la fabricación de los especímenes de control se utilizó como refuerzo una malla de alambre electrosoldado laminado en frío con esfuerzos de fluencia y de ruptura, respectivamente, de 490 M Pa y 560 M Pa, según datos especificados por el proveedor; no se consideró necesaria su verificación experimental ya que es un producto comercial sujeto a estrictas normas de control de resistencia y fabricados bajo las $\mathrm{N}$ ormas $\mathrm{O}$ ficiales Mexicanas N MX B-253 y N M X B-290.

\subsection{Acero inoxidable}

El acero inoxidable utilizado en la fabricación de cospeles, y por ende como refuerzo en los especímenes de concreto objeto de este estudio, es el designado por la AISI como tipo 430 , acero ferrítico con un $16 \%$ a $18 \%$ de cromo. A fin de determinar la respuesta del material en condiciones de carga para establecer las relaciones que rigen su comportamiento y su grado de ductilidad se efectuaron ensayes de tracción en probetas de acero inoxidable en distintos espesores de conformidad con los procedimientos que señalan las normas ASTM A370 (ASTM, 2007) y ASTM E8 (ASTM, 2004) (Figura 2).

Se ensayaron un total de 249 especímenes en diversos espesores que van desde $0.29 \mathrm{~mm}$ hasta 2.97 $\mathrm{mm}$, obteniéndose en todos los casos los valores de esfuerzos y deformaciones hasta la falla, el límite de fluencia, el esfuerzo a la ruptura y el porcentaje de alargamiento. El valor medio así obtenido para el límite de fluencia resultó ser de 332 M Pa y de 489 MPa para el esfuerzo a la ruptura, con valores confiables de acuerdo a procedimientos estadísticos basados en distribuciones normales $(\mathrm{ACl}, 2002)$ de $302 \mathrm{MPa}$ y de $462 \mathrm{MPa}$ respectivamente. Si se compara con el esfuerzo de fluencia mínimo especificado para la malla de alambre electrosoldado de 490 M Pa y su valor de ruptura de 560 $\mathrm{MPa}$, resulta evidente la necesidad de emplear cuantías mayores de refuerzo para compensar la baja resistencia del material. 


\section{Programa de pruebas}

\subsection{Concreto hidráulico}

Se elaboraron un total de 26 especímenes reforzados tanto con malla de acero inoxidable como con malla de alambre electrosoldado a fin de determinar su capacidad de carga. Por cada uno de dichos especímenes se obtuvieron dos cilindros de concreto a partir de la misma mezcla, haciendo un total de 52. Las muestras fueron preparadas de conformidad con el "M étodo de Fabricación y Curado en el Laboratorio de Muestras de Prueba" de la norma ASTM C192 (ASTM, 2007) y ensayadas a una edad de 28 días de acuerdo con el "M étodo de Prueba para Determinar la Resistencia a la Compresión de Cilindros Moldeados de Concreto" de la norma ASTM C39 (ASTM, 2005). A partir de los datos de resistencia de las pruebas individuales se obtuvo un valor promedio $\mathrm{f}_{\mathrm{cr}}$ de $22.8 \mathrm{MPa}$ y un valor confiable para la resistencia de diseño f'c de 19.2 $\mathrm{MPa}$.
Para éste análisis experimental fueron elegidos dos tipos de malla como refuerzo, la primera de ellas con un espesor de $1.15 \mathrm{~mm}, 305 \mathrm{~mm}$ de ancho efectivo y perforaciones de $17 \mathrm{~mm}$ de diámetro, mientras que el segundo tipo de malla elegida cuenta con un espesor de $1.43 \mathrm{~mm}$, ancho de $152 \mathrm{~mm}$ y perforaciones con diámetro de $21 \mathrm{~mm}$. Paralelamente se elaboraron especímenes de control reforzados convencionalmente, para fines comparativos, con malla de alambre electrosoldado estirado en frío, con un esfuerzo de fluencia mínimo de $490 \mathrm{M} \mathrm{Pa}$, con tres alambres calibre 6 de $4.88 \mathrm{~mm}$ de diámetro en el sentido longitudinal, con una separación de $152 \mathrm{~mm}$ a centros. Los especímenes ensayados constituyen prototipos a escala natural pues cuentan con un claro libre similar a los que usualmente se manejan en vivienda popular. La Figura 3 muestra de manera esquemática tanto la geometría como la posición del acero de refuerzo.

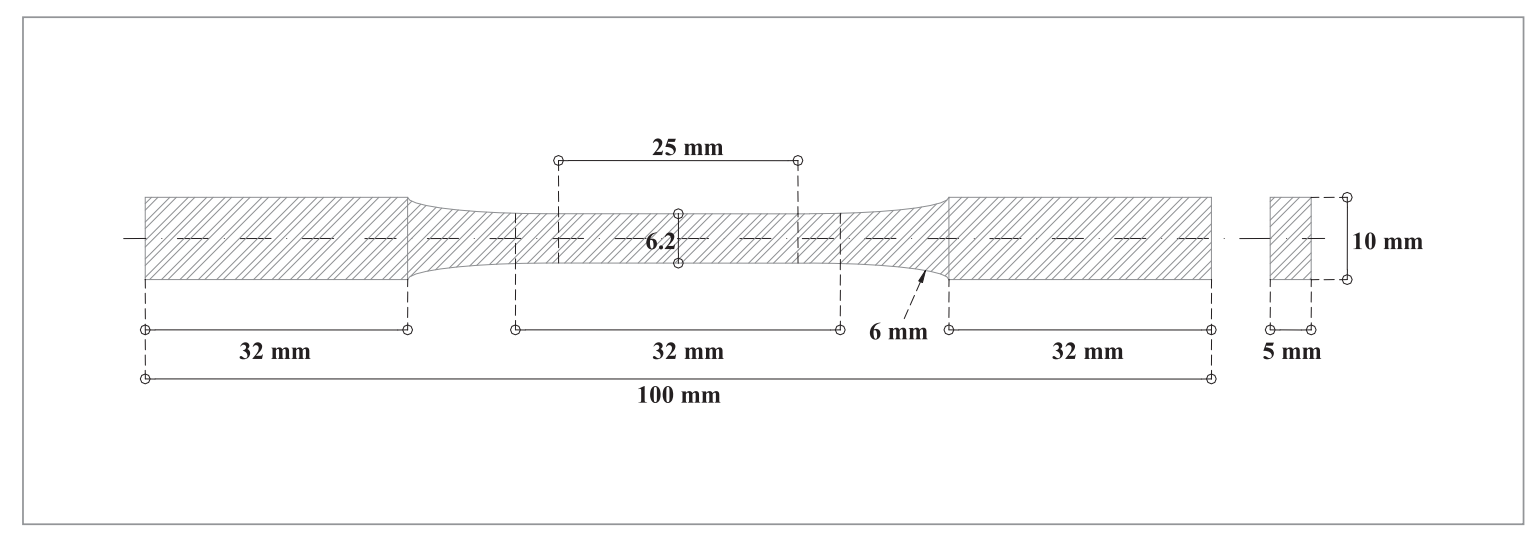

Figura 2. Esquema de las probetas de tracción

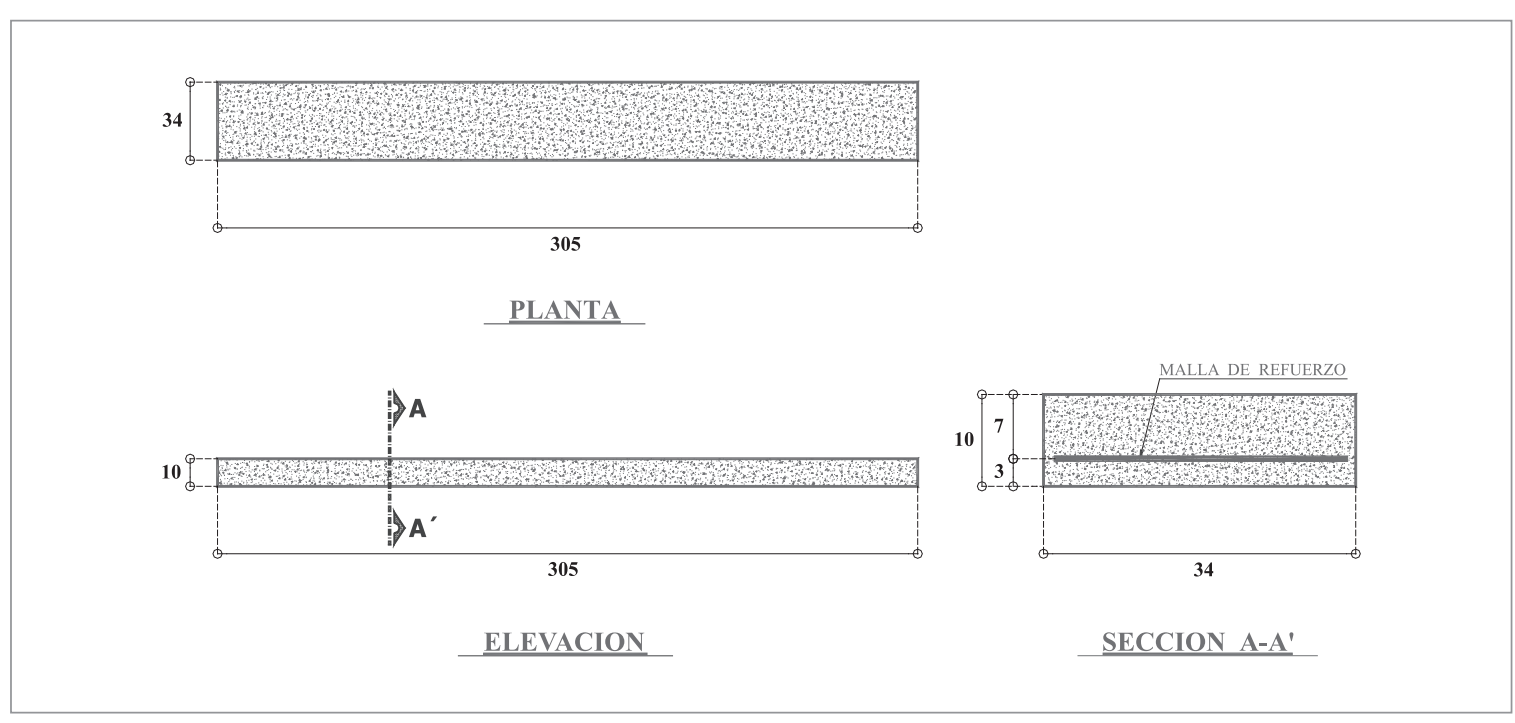

Figura 3. Geometría y refuerzo de especímenes (acotaciones en $\mathrm{mm}$ ) 
La Tabla 1 muestra el número de especímenes ensayados. La malla de cospeles de $1.43 \mathrm{~mm}$ de espesor cuenta con un ancho de $152 \mathrm{~mm}$, por lo que debieron colocarse dos mallas de este tipo a fin de complementar el refuerzo en todo el ancho del espécimen; en el caso de aquellos reforzados con tres mallas, la tercera se colocó únicamente en el tercio medio del espécimen.

Tabla 1. Programa de pruebas

\begin{tabular}{||c|c||}
\hline \multicolumn{1}{|c||}{ Tipo de refuerzo } & Especímenes ensayados \\
\hline $\begin{array}{l}\text { 1) } 1 \text { Malla de cospeles de } 1.15 \mathrm{~mm} \text { de } \\
\text { espesor y } 305 \mathrm{~mm} \text { de ancho en un solo } \\
\text { lecho }\end{array}$ & 5 \\
\hline $\begin{array}{l}\text { 2) } 2 \text { Mallas de cospeles de } 1.43 \mathrm{~mm} \text { de } \\
\text { espesor y } 152 \mathrm{~mm} \text { de ancho en un solo } \\
\text { lecho }\end{array}$ & 5 \\
\hline $\begin{array}{l}\text { 3) } 3 \text { Mallas de cospeles de } 1.43 \mathrm{~mm} \text { de } \\
\text { espesor y } 152 \mathrm{~mm} \text { de ancho en dos } \\
\text { lechos }\end{array}$ & 3 \\
\hline 4) 1 Malla de alambre electrosoldado de \\
3 alambres de $4.88 \mathrm{~mm}$ de diámetro
\end{tabular}

Las pruebas de carga consistieron en la aplicación de una sola carga concentrada al centro del claro, de manera monotónica desde un valor nulo hasta el que produjo el colapso de la viga, con una velocidad de movimiento del cabezal en un rango comprendido entre $0.80 \mathrm{~mm} / \mathrm{min}$, y $1.00 \mathrm{~mm} / \mathrm{min}$. Los especímenes fueron ensayados en la totalidad de los casos a la edad de 28 días en una Máquina U niversal de Pruebas marca Forney con capacidad de $1500 \mathrm{KN}$ y sensibilidad de 50 $\mathrm{N}$; las lecturas de deformación fueron tomadas a partir del registro de micrómetros de carátula con sensibilidad de una milésima de pulgada colocados en el centro del claro de los modelos. En la Figura 4 se muestra el esquema de carga aplicado, mientras que en la fotografía de la Figura 5 se puede apreciar el montaje de uno de los especímenes en la máquina de pruebas.

El comportamiento de la viga fue verificado a partir de los niveles de carga aplicados y de las deformaciones medidas al centro del claro; el nivel de agrietamiento de las vigas reforzadas con la malla de cospeles, comparativamente con el de los especímenes de referencia, así como el tipo de falla, se tomaron en consideración únicamente de manera cualitativa.

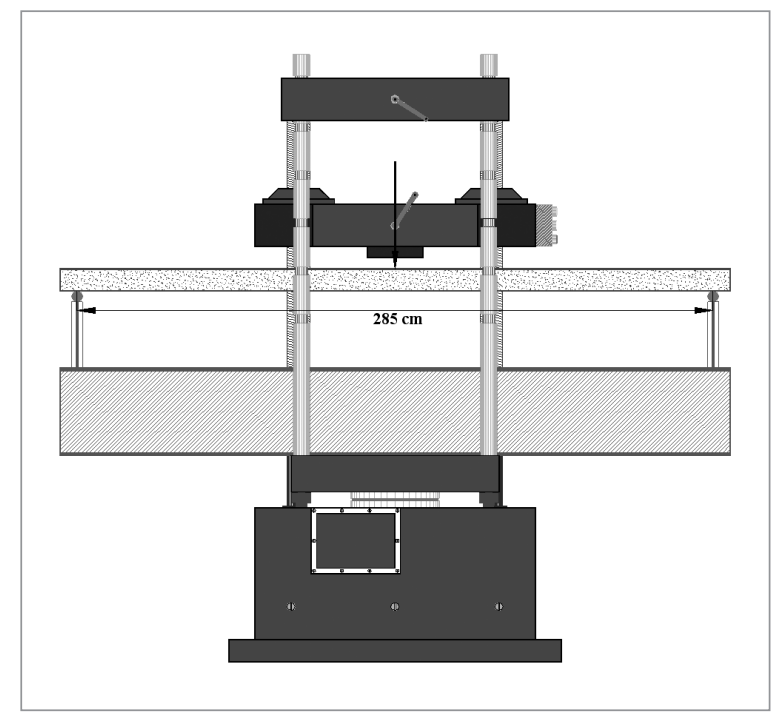

Figura 4. Esquema de carga

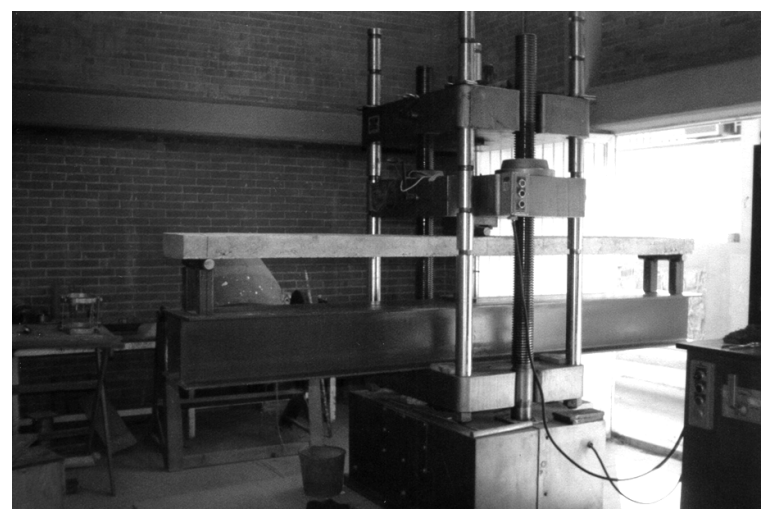

Figura 5. M ontaje de uno de los especímenes en la máquina de pruebas

\section{Resultadosy discusión}

Es posible realizar un primer análisis comparativo entre los especímenes con distinto tipo de refuerzo a través de su comportamiento expresado mediante curvas Carga-Deflexión, que en promedio por tipo de espécimen se muestran en la Figura 6. Desde un punto de vista meramente cualitativo es evidente el pobre desempeño que representa el comportamiento de los especímenes reforzados con la malla de cospeles de $1.15 \mathrm{~mm}$, tanto en lo que se refiere a la capacidad de carga como a la ductilidad desarrollada, tomando no sólo como referencia los especímenes de control con refuerzo convencional a base de malla electrosoldada, sino los que se reforzaron con malla de cospeles de $1.43 \mathrm{~mm}$, cuyo comportamiento representa una notable mejoría. 


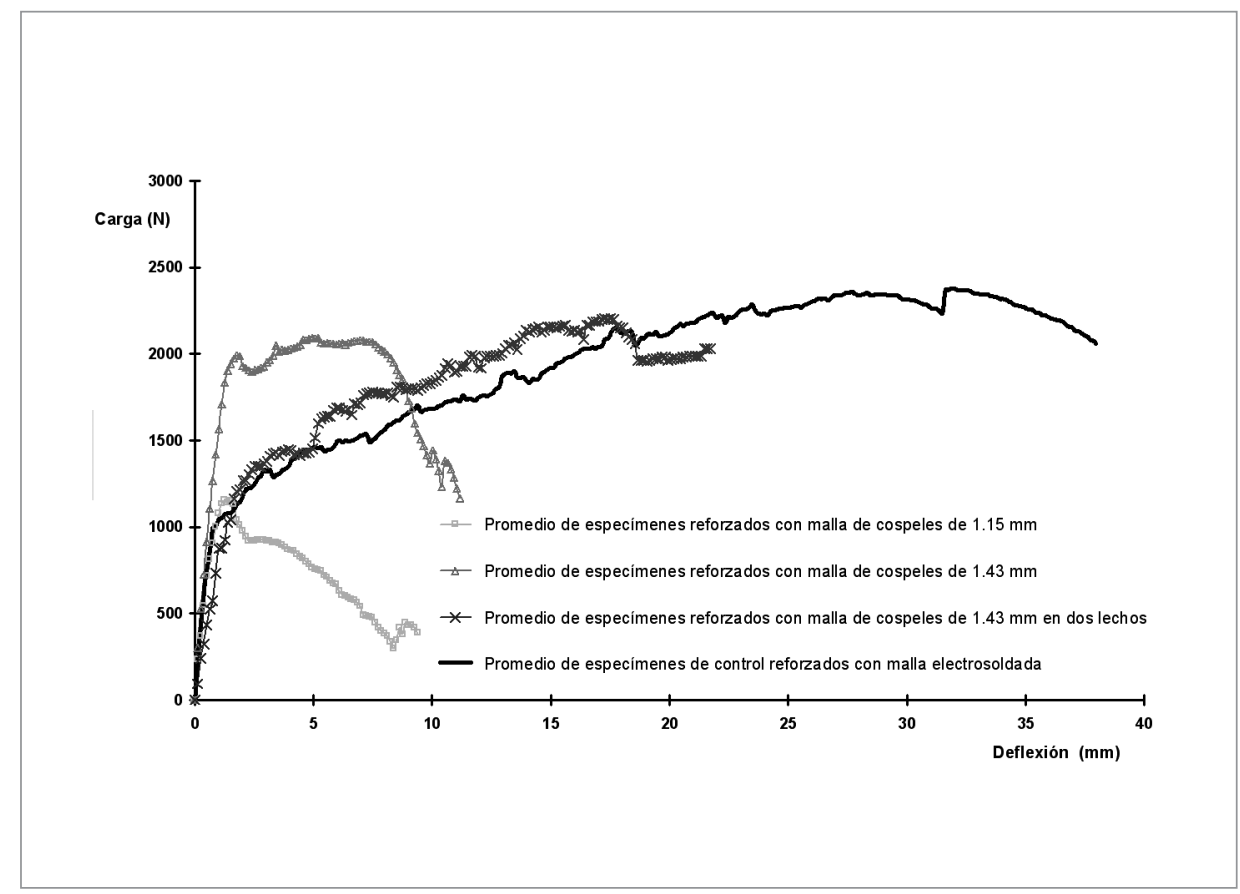

Figura 6. Curvas promedio Carga - Deflexión al centro del claro

Siguiendo estos criterios existiría una natural inclinación a desechar los especimenes con tal refuerzo, por constituir éste una cantidad probablemente muy por debajo de la cuantía mínima requerida.

Los especímenes reforzados con malla de cospeles de $1.43 \mathrm{~mm}$ tuvieron un comportamiento más acorde con el que cabría esperar a partir de la evidencia representada por los prototipos de referencia, en especial aquellos en los que se colocó una tercera malla. Como se puede apreciar en el gráfico de la figura 6, ambos tipos de vigas alcanzaron resistencias similares a la de los especímenes con refuerzo convencional.

En la Tabla 2 se han reproducido los valores obtenidos relativos al comportamiento de los prototipos ensayados. Los parámetros de dicho comportamiento susceptibles de comparación se refieren por una parte a las máximas deformaciones alcanzadas y el grado de ductilidad desarrollado, definido este como el cociente entre la deflexión máxima y la deflexión elástica al inicio de la fluencia, y por la otra a la capacidad resistente del elemento en términos de una carga uniforme equivalente por unidad de superficie, calculada esta última mediante la igualación del momento flexionante que esta produciría con aquel provocado por la carga de falla; sin embargo, una tercera fuente de datos que pueden resultar decisivos al momento de desechar algunos de los especímenes se consignan en la última columna de la tabla, y son los que corresponden al tipo de falla del elemento y a su respuesta en condiciones cercanas al colapso.

Atendiendo a lo anterior, parece claro y definitivo que no sólo los especímenes reforzados con malla de $1.15 \mathrm{~mm}$, como se comentó líneas arriba, sino también aquellos que se reforzaron con doble malla de $1.43 \mathrm{~mm}$ en un solo lecho, deberán ser descartados como alternativas viables en sistemas de cubierta ya que el carácter frágil de su comportamiento en la cercanía de la falla resulta inadmisible. Esto puede ser confirmado si se observan los resultados relativos al grado de deformación alcanzado por los especímenes en cuestión en las columnas 2 a 4 y se comparan con los parámetros de referencia en el último renglón de la tabla, siendo éstos últimos del orden de casi cuatro veces el valor de aquellos en el caso de las deflexiones máximas alcanzadas, y casi cinco veces en lo que respecta a la ductilidad, si bien es cierto que la capacidad resistente de los especímenes con malla de $1.43 \mathrm{~mm}$ ( $2173 \mathrm{~N}$ en promedio) alcanzó un $90 \%$ de la de los especímenes de control $(2419 \mathrm{~N})$. 
Tabla 2. Resultados comparativos de las pruebas de carga

\begin{tabular}{|c|c|c|c|c|c|c|c|}
\hline $\begin{array}{l}\text { Tipo de } \\
\text { refuerzo }\end{array}$ & $\begin{array}{c}\text { Deflexión } \\
\text { elástica } \\
(\mathrm{mm})\end{array}$ & $\begin{array}{c}\text { Deflexión } \\
\text { máxima } \\
(\mathrm{mm})\end{array}$ & \begin{tabular}{|c|} 
Coef. \\
de \\
Ductilidad
\end{tabular} & $\begin{array}{c}\text { Carga } \\
\text { máx. } \\
(\mathrm{N})\end{array}$ & $\begin{array}{c}\text { Mom. } \\
\text { Flex. } \\
\text { (N-m) }\end{array}$ & $\begin{array}{c}\omega_{\mathrm{eq}}{ }^{*} \\
\left(\mathrm{~N} / \mathrm{m}^{2}\right)\end{array}$ & $\begin{array}{c}\text { Tipo de } \\
\text { Falla }\end{array}$ \\
\hline $\begin{array}{l}1 \text { Malla de } \\
\text { cospeles de } 1.15 \\
\text { mm de espesor } \\
\text { y } 305 \mathrm{~mm} \text { de } \\
\text { ancho en un } \\
\text { lecho }\end{array}$ & $\begin{array}{c}1.30 \\
(99 \%)^{\star \star}\end{array}$ & $\begin{array}{c}9.40 \\
(25 \%)\end{array}$ & $\begin{array}{c}7.73 \\
(22 \%)\end{array}$ & $\begin{array}{c}1216 \\
(50 \%)\end{array}$ & $\begin{array}{c}867 \\
(50 \%)\end{array}$ & $\begin{array}{c}2511 \\
(50 \%)\end{array}$ & $\begin{array}{l}\text { Frágil } \\
\text { Una sola } \\
\text { grieta al } \\
\text { centro del } \\
\text { claro. }\end{array}$ \\
\hline $\begin{array}{l}2 \text { Mallas de } \\
\text { cospeles de } 1.43 \\
\text { mm de espesor } \\
\text { y } 152 \mathrm{~mm} \text { de } \\
\text { ancho en un } \\
\text { lecho }\end{array}$ & $\begin{array}{c}1.60 \\
(122 \%)\end{array}$ & $\begin{array}{l}11.20 \\
(29 \%)\end{array}$ & $\begin{array}{l}7.04 \\
(20 \%)\end{array}$ & $\begin{array}{l}2173 \\
(90 \%)\end{array}$ & $\begin{array}{c}1548 \\
(90 \%)\end{array}$ & \begin{tabular}{|c|}
4483 \\
$(90 \%)$
\end{tabular} & $\begin{array}{l}\text { Frágil } \\
\text { Una sola } \\
\text { grieta al } \\
\text { centro del } \\
\text { claro. }\end{array}$ \\
\hline $\begin{array}{l}3 \text { Mallas de } \\
\text { cospeles de } 1.43 \\
\text { mm de espesor } \\
\text { y } 152 \mathrm{~mm} \text { de } \\
\text { ancho en dos } \\
\text { lechos }\end{array}$ & $\begin{array}{c}2.16 \\
(165 \%)\end{array}$ & $\begin{array}{l}21.72 \\
(57 \%)\end{array}$ & $\begin{array}{l}10.20 \\
(27 \%)\end{array}$ & $\begin{array}{c}2260 \\
(93 \%)\end{array}$ & $\begin{array}{c}1611 \\
(93 \%)\end{array}$ & \begin{tabular}{|c|}
3502 \\
$(70 \%)$
\end{tabular} & $\begin{array}{l}\text { Dúctil } \\
\text { Grietas en } \\
\text { el tercio } \\
\text { central del } \\
\text { claro. }\end{array}$ \\
\hline $\begin{array}{l}1 \text { Malla de } \\
\text { alambre } \\
\text { electrosoldado } \\
\text { de } 3 \text { alambres } \\
\text { de } 4.88 \mathrm{~mm} \text { de } \\
\text { diámetro }\end{array}$ & $\begin{array}{c}1.31 \\
(100 \%)\end{array}$ & $\begin{array}{c}38.00 \\
(100 \%)\end{array}$ & $\begin{array}{c}34.40 \\
(100 \%)\end{array}$ & $\begin{array}{c}2419 \\
(100 \%)\end{array}$ & $\begin{array}{c}1724 \\
(100 \%)\end{array}$ & \begin{tabular}{|c|}
4993 \\
$(100 \%$
\end{tabular} & $\begin{array}{l}\text { Dúctil } \\
\text { Grietas a } \\
\text { todo lo } \\
\text { largo de la } \\
\text { viga. }\end{array}$ \\
\hline
\end{tabular}

Los tres especímenes reforzados cada uno con tres mallas de cospeles de $1.43 \mathrm{~mm}$ de espesor, fueron fabricados con la intención de mejorar las características de la respuesta estructural en términos de la ductilidad desarrollada al momento del colapso; se colocaron dos mallas de $152 \mathrm{~mm}$ de ancho lado con lado en un solo lecho y la tercera malla en un segundo lecho, directamente por encima del primero; ésta última únicamente se colocó en el tercio medio de la viga. El patrón de agrietamiento difiere significativamente con los anteriores al presentarse grietas prácticamente en todo el tercio medio del claro libre de la viga, como se pueden apreciar en la fotografía de la Figura 7, siendo este de una forma mucho más dúctil y presentándose la falla, en la totalidad de los casos, a un tercio del apoyo.

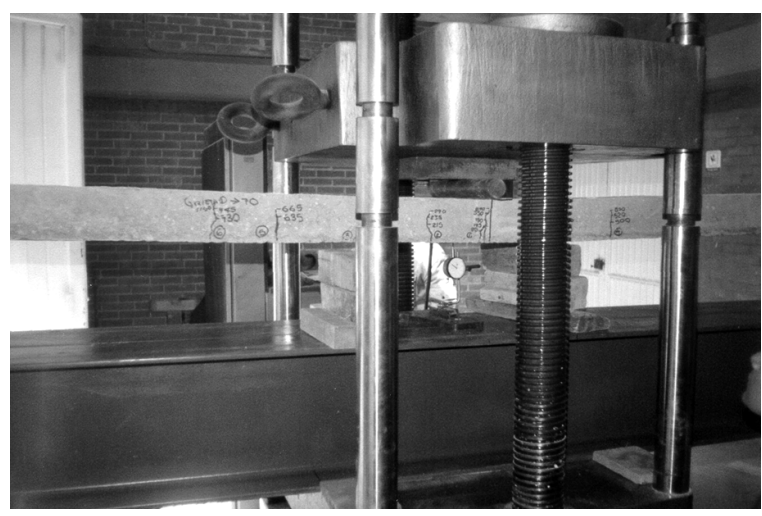

Figura 7. Patrón de agrietamiento en el tercio central
La forma en que se presenta la falla en estos especímenes resulta ser significativa, toda vez que señala las directrices en que debe ser evaluada la capacidad de carga de los mismos; de haber ocurrido la falla al centro del claro, la capacidad de carga uniforme equivalente por unidad de superficie podría calcularse conforme al criterio seguido para los dos tipos de especímenes anteriormente descritos, esto es, igualando los momentos producidos en la sección central y despejando el valor de $\omega$ sin embargo, el momento flexionante que produce el colapso de la viga no es, a diferencia de los casos anteriores, el máximo que se presenta al centro del claro, sino un valor menor, en el tercio del claro, con lo que ahora la carga uniforme equivalente será la que genere dicho valor de momento en dicha sección transversal, valor que, como se puede apreciar en la Tabla 2, es significativamente menor.

Considerando lo anterior, la capacidad portante registrada en promedio de los elementos ensayados, convertida a una carga uniforme equivalente de $3502 \mathrm{~N} / \mathrm{m}^{2}$, se encuentra aún por debajo de la de los especímenes de referencia establecida en $4993 \mathrm{~N} / \mathrm{m}^{2}$, pues aquella representa apenas un $70 \%$ del valor de ésta última. Esta sobrecarga útil (adicional al peso propio de la viga) deberá estar compuesta tanto por la carga muerta debida a elementos de recubrimiento, acabados, etc., como por la carga viva atribuible al destino de la 
construcción (en esta caso vivienda); para fines de diseño, en ellas deberán ser consideradas las incertidumbres propias de su naturaleza aleatoria a través de los factores de carga apropiados cuyos valores, de acuerdo al código del Instituto Americano del Concreto ( $\mathrm{ACl}, 2005)$, han sido fijados en 1.2 y 1.6 respectivamente para las carga muertas y vivas, de tal suerte que la carga resistente puede ser expresada de la forma:

$$
\omega_{U}=\gamma_{C M} * \omega_{C M}+\gamma_{C V}{ }^{*} \omega_{C V}
$$

Si de acuerdo también con los códigos de diseño asumimos un valor para la carga viva de 1700 $\mathrm{N} / \mathrm{m}^{2}$ (correspondiente a cubiertas destinadas para vivienda) es posible determinar la carga muerta disponible en base a los resultados obtenidos:

$$
\begin{aligned}
& \omega_{C M}=\frac{\omega_{U}-\gamma_{C V} * \omega_{C V}}{\gamma_{C M}} \\
& \omega_{C M}=\frac{3502 \mathrm{~N} / \mathrm{m}^{2}-1.6 *\left(1700 \mathrm{~N} / \mathrm{m}^{2}\right)}{1.2} \\
& \omega_{C M}=652 \mathrm{~N} / \mathrm{m}^{2}
\end{aligned}
$$

valor éste último que parece suficiente para considerarse como sobrecarga muerta debida al peso de acabados y recubrimientos, adicional al propio peso de la losa.

Finalmente, merece un llamado de atención el hecho de que la capacidad de carga promedio de los especímenes reforzados con malla de $1.15 \mathrm{~mm}$ represente apenas la mitad del valor correspondiente de una viga de concreto simple. Como puede observarse en la sexta columna de la tabla 2, el momento flexionante de falla de los especímenes reforzados con malla de cospeles de $1.43 \mathrm{~mm}$ alcanzó valores del orden de magnitud del momento de agrietamiento (cuyo valor es de $1603 \mathrm{~N}$ $\mathrm{m}$, calculado como el momento que resistiría la viga de no existir ningún tipo de refuerzo), y las vigas de referencia obtuvieron un valor de $1724 \mathrm{~N} \mathrm{~m}$, ligeramente superior, lo cual significa que tanto el refuerzo de cospeles utilizado como la malla electrosoldada representan la mínima cantidad de refuerzo con el que es posible proveer una viga de $10 \mathrm{~cm}$. de peralte, mientras que el refuerzo a base de cospeles de $1.15 \mathrm{~mm}$ cuenta con un área de acero muy por debajo de dicho valor mínimo (lo que corrobora la tesis ya expresada aquí en cuanto a desechar éste tipo de refuerzo en elementos estructurales bajo esfuerzos de flexión). ¿Por qué entonces la resistencia de éstos elementos resulta ser inferior a la de una viga de concreto simple? ¿Acaso no cabría esperar una capacidad de carga que al menos iguale éste valor? Resulta difícil aventurar una respuesta que justifique ambos cuestionamientos sin dejar lugar a dudas; es posible que la presencia de una fuerte discontinuidad representada por la malla de refuerzo dentro de la matriz de concreto, sin que ésta contribuya de manera apreciable en la resistencia del elemento, aunada a una pobre adherencia de ambos materiales, ceda lugar a una alta concentración de esfuerzo que conduzca a pequeñas fracturas microscópicas cuya propagación origine una falla prematura del espécimen; esto sólo es una hipótesis que deberá ser evaluada y ser objeto de posteriores investigaciones.

\section{Conclusiones}

A partir de los resultados mostrados y su discusión y análisis es posible definir las siguientes conclusiones sobre el comportamiento en condiciones de falla del tipo de elementos estudiado:

- Los especímenes con refuerzo a base de malla de acero inoxidable de $1.15 \mathrm{~mm}$ de espesor obtuvieron una muy pobre respuesta en comparación con los especímenes a base de refuerzo tradicional en términos de resistencia y ductilidad, razón por la cual este tipo de refuerzo no puede ser recomendable para elementos que trabajen bajo fuertes solicitaciones de flexión.

- Los especímenes con refuerzo a base de malla de acero inoxidable de $1.43 \mathrm{~mm}$ de espesor tuvieron un mejor desempeño. Su resistencia promedio alcanzó niveles del orden del $90 \%$ de la capacidad alcanzada por los especímenes de control. A pesar de lo promisorio de estos últimos resultados observados en los especímenes reforzados con malla de acero inoxidable de $1.43 \mathrm{~mm}$ de espesor, la relativamente baja ductilidad desarrollada aunada al tipo de falla de naturaleza frágil denota aún un comportamiento inadmisible, por lo que, tratando de mejorar las características de su respuesta, fueron fabricados tres especimenes con una tercera malla adicional en el tercio medio del claro, cuyo comportamiento se resumen aquí: 
- La capacidad de carga se mantuvo en niveles similares a los especímenes anteriores.

- La ductilidad desarrollada alcanzó valores del orden de la mitad de la de los especímenes de control, lo que representa una notable mejoría. Los patrones de agrietamiento y deformación, de naturaleza dúctil, son similares a los que se generaron en las vigas con refuerzo tradicional.

- El mecanismo de colapso generado en la totalidad de las vigas provistas con este tipo de refuerzo, con la formación de una articulación plástica al tercio del claro, donde se interrumpe la tercera malla de refuerzo, presupone la aparición de un tipo de falla prematura, toda vez que el momento flexionante que se genera en dicha sección de falla es menor que el correspondiente al centro del claro; surge entonces la duda de por qué en ambos tipos de especímenes la falla ocurre en una sección con la misma cantidad de refuerzo pero bajo diferentes niveles de esfuerzo; la respuesta a tal cuestionamiento requiere de una mayor evidencia experimental.

Con todo lo anterior, es concluyente la factibilidad del uso del material de desecho empleado en la fabricación de moneda de acero inoxidable como refuerzo de tensión en losas de claros pequeños; como se ha visto, éste tipo de refuerzo representa una alternativa al uso de la malla electrosoldada a un precio razonablemente competitivo.

\section{Referencias}

ACl Standard 214R-02 (2002), "Evaluation of Strength Test Results of Concrete", American Concrete Institute, Farmington Hills, MI, USA.

ACI Standard 318-05/318R-05 (2005), “Building Code Requirements for Structural Concrete and Commentary", American Concrete Institute, Farmington Hills, MI, USA.

ASTM Standard A370-07b (2007), "Standard Test Methods and Definitions for Mechanical Testing of Steel Products", American Society for Testing and Materials, West Conshohocken, PA, USA. ASTM Standard A955/A955M-96 (1996), "Standard Specification for Deformed and Plain Stainless Steel Bars for Concrete Reinforcement", American Society for Testing and Materials, West Conshohocken, PA, USA.
ASTM Standard C39/C39M-05e1 (2005), "Standard Test Method for Compressive Strength of Cylindrical Concrete Specimens", American Society for Testing and Materials, West Conshohocken, PA, USA. ASTM Standard C192/C192M-07 (2007), "Standard Practice for Making and Curing Concrete Test Specimens in the Laboratory", American Society for Testing and Materials, West Conshohocken, PA, USA. ASTM Standard E8-04 (2004), "Standard Test Methods for Tension Testing of Metallic M aterials", American Society for Testing and Materials, West Conshohocken, PA, USA.

Baltazar M., Almeraya F., N ieves D., Borunda A., Maldonado E., O rtiz A. (2007), "Corrosión del acero inoxidable 304 como refuerzo en concreto expuesto a cloruros y sulfatos", Scientia et Technica, 13(36), 353-357, Pereira, Risaralda, Colombia.

BA 84/02 (2002), Design M anual for Roads and Bridges, Vol. 1, Sec 3, Parte 15: "Use of stainless steel reinforcement in highway structures", The Highways Agency, U.K.

BS 6744:2001 (2001), "Stainless steel bars for the reinforcement of and use in concrete requirements and test methods", British Standards Institution, Londres, U.K..

Castro-Borges P., Troconis de Rincón O., Moreno E.I., Torres-Acosta A.A., M artínez-Madrid M., Knudsen A.

(2002), "Performance of a 60-year old concrete pier of stainless steel reinforcement", M aterials Performance, 41(10), 50-55, Houston, USA.

Concrete Society (1998), "Guidance on the use of stainless steel reinforcement", 1a edición, 56 pp, Surrey, U.K.

Hunkeler F. (2000), "Einsatz von nichtrostenden Bewehrungsstählen im Betonbau" (Use of non-corroding Reinforcing Steels in Concrete Construction), (en línea), Eidgenössisches Departement für Umwelt, Verkehr, Energie und Kommunikation/Bundesamt für Strassen, Switzerland, http://www.tfb.ch/htdocs/Files/ VSS/VSS\% 20543\% 20englisch.pdf. Acceso: junio 2008.

Li C.Q., Melchers R.E., Lawanwisut W. (2005), "Vulnerability assessment of corrosion-affected concrete structures", Magazine of Concrete Research, 57(9), 557, Londres, UK.

Medina-Romero L. (2006), “Análisis de la viabilidad económica y ambiental de armaduras corrugadas de 
acero inoxidable en elementos de hormigón armado sometidos a clases de exposición agresivas. Aplicación a elementos en contacto con aguas residuales agresivas", Tesis de licenciatura, U niversidad Politécnica de Cataluña, Escuela Técnica Superior de Ingeniería de Caminos, Canales y Puertos de Barcelona, Barcelona, España.

Moreno E.I., Torres-Acosta A.A., Castro-Borges P. (2004), "Construcción del muelle de Progreso", Ingeniería Revista Académica, 8(1), 61-66, Mérida, Yucatán, México.

Torres-Acosta A.A., Pérez-Q uiroz J.T., Ramírez-Rentería A.J., Martínez.Madrid M. (2006), “Estudio de la corrosión en barras de acero inoxidable en concreto contaminado por cloruros cuando se le aplican esfuerzos residuales", Publicación Técnica No 287, 1-10, Secretaria de Comunicaciones y Transportes, Instituto Mexicano del Transporte, Sanfandila, Q ro., México. 